\title{
CONTRIBUIÇÕES DA ENFERMAGEM OBSTÉTRICA PARA AS BOAS PRÁTICAS NO TRABALHO DE PARTO E PARTO VAGINAL
}

Taynara Cassimiro de Moura Alves ${ }^{1}$ Amanda Santos Fernandes Coelho ${ }^{1}$ Marilia Cordeiro de Sousa ${ }^{2}$

Nayara Franklin Cesar ${ }^{1}$

Priscila Salomão da Silva

Leonora Rezende Pacheco²

\author{
https://orcid.org/0000-0002-0312-934X \\ https://orcid.org/0000-0001-5379-2740 \\ https://orcid.org/0000-0001-6643-2365 \\ https://orcid.org/0000-0003-3857-554X \\ https://orcid.org/0000-0001-9511-7444 \\ https://orcid.org/0000-0001-6048-3911
}

Objetivo: analisar as contribuições da enfermagem obstétrica para as boas práticas no trabalho de parto e parto vaginal. Metodologia: estudo transversal, quantitativo, retrospectivo, envolvendo 475 prontuários de mulheres com gestação de risco habitual, do estado de Goiás, no período de janeiro a dezembro de 2016. Análise com testes de qui-quadrado de Yates e Exato de Fisher. Resultados: houve associação entre os partos sem os enfermeiros residentes em obstétrica e a não utilização dos métodos não farmacológicos para alívio da dor ( $p<0.0000001)$, não utilização do partograma ( $<<0.0000001)$, ausência de acompanhante no parto ( $p<0.0000001$ ), clampeamento precoce do cordão umbilical ( $p=0.00004323$ ), e a privação da amamentação na primeira hora $(p=0.0001509)$. Já os partos assistidos por enfermeiros residentes em obstetrícia associaramse à não realização da episiotomia ( $p<0.0000001)$. Conclusão: enfatiza-se a importância da atuação da Enfermagem Obstétrica no parto para garantir assistência humanizada e boas práticas.

Descritores: Parto Normal; Parto Humanizado; Enfermagem Obstétrica; Assistência Perinatal.

\section{CONTRIBUTIONS OF THE OBSTETRICAL NURSING FOR THE GOOD PRACTICES IN LABOR AND VAGINAL DELIVERY}

Objective: To analyze the contributions of the obstetrical nursing for the good practices in labor and vaginal delivery. Methodology: A cross-sectional, quantitative and retrospective study involving 475 medical records of women with normal risk pregnancies, in Goias State, during the period from January to December of 2016. Analysis using Yates chi square test and Fisher's Exact test. Results: There was an association between the labors without the obstetric nursing residents and the nonuse of non-pharmacological methods for providing pain relief ( $p<0.0000001)$, non-use of partograph ( $p<0.0000001)$, absence of companionship during labour ( $p<0.0000001$ ), early clamping of the umbilical cord ( $p=0.00004323$ ), and to the deprivation of breastfeeding in the first hour ( $p=0.0001509)$. The deliveries assisted by obstetric nursing residents were associated to the nonperformance of the episiotomy ( $p<0.0000001$ ). Conclusion: Emphasis is placed on the importance of obstetric nursing work in childbirth to ensure humanized care and good practices.

Descriptors: natural childbirth, humanizing delivery, obstetric nursing, perinatal care.

\section{CONTRIBUCIONES DE LA ENFERMERİA OBSTÉTRICA PARA LAS BUENAS PRÁCTICAS EN EL TRABAJO DE PARTO Y PARTO VAGINAL}

Objetivo: Analizar las contribuciones de la enfermería obstétrica para las buenas prácticas en el trabajo de parto y parto vaginal. Metodología: Estudio transversal, cuantitativo, retrospectivo, involucrando 475 prontuarios de mujeres con gestación de riesgo habitual, del Estado de Goiás, en el período de enero a diciembre de 2016. Análisis con pruebas de chi-cuadrado de Yates y Exacto de Fisher. Resultados: Se observó asociación entre los partos sin los enfermeros residentes en obstétrica y la no utilización de los métodos no farmacológicos para alivio del dolor ( $p<0.0000001$ ), no utilización del partograma ( $p<0.0000001$ ), ausencia de acompañante en el parto ( $p<0.0000001)$, pinzamiento precoz del cordón umbilical ( $p=0.00004323$ ), y la privación de la lactancia en la primera hora ( $p=0.0001509)$. Los partos asistidos por enfermeros residentes en obstetricia se asociaron a la no realización de la episiotomía ( $<$ <0.0000001). Conclusión: Se enfatiza la importancia de la actuación de la enfermería obstétrica en el parto para garantizar asistencia humanizada y buenas prácticas.

Descriptores: Parto normal; Parto Humanizado; Enfermería Obstétrica; Atención Perinatal.

${ }^{1}$ Hospital Materno-Infantil da Secretaria de Estado da Saúde de Goiás.

2Universidade Federal de Goiás-UFG.

Autor correspondente: Amanda Santos Fernandes Coelho. E-mail: amandasantospi@yahoo.com.br 


\section{INTRODUÇÃO}

O parto é um evento fisiológico e natural, constituindo experiência única à mulher e sua família, por isso deve ser vivenciado com segurança, dignidade e beleza ${ }^{(1)}$. As boas práticas de atenção ao parto e nascimento são ações benéficas e protetoras à vida da mulher e do recém-nascido que certamente impactam diretamente na redução da morbimortalidade materna e neonatal. A não adoção dessas práticas pode ser considerada negligência ou iatrogenia no serviço em saúde, pois coloca em risco desnecessário a mulher e o bebê( ${ }^{(2)}$.

O Ministério da Saúde (MS), para em melhorar a qualidade da assistência por meio da humanização do serviço, criou o Programa de Humanização no Pré-natal e Nascimento, e ainda tem estimulado um movimento institucional, político, ético e afetivo em prol de trabalho conjunto, por meio da criação de políticas e diretrizes em favor da humanização e boas práticas no atendimento à mulher em seu ciclo reprodutivo ${ }^{(3,4)}$.

A participação do enfermeiro obstétrico no cenário do parto pode favorecer o equilíbrio entre o processo fisiológico da parturição e as intervenções necessárias, reconhecendo e corrigindo os desvios da normalidade, e encaminhando aquelas que demandem assistência especializada, de forma a dispensar maior cuidado individualizado e personalizado para cada mulher e sua família ${ }^{(4.5)}$. Com isso, o MS, por meio da Rede Cegonha, incentiva a formação e atuação de enfermeiras obstétricas, para um atendimento mais humanizado e direcionado pelas boas práticas ${ }^{(6)}$.

A enfermagem obstétrica tem ganhado espaço nas políticas públicas de saúde devido ao seu olhar qualificado e humanizado do processo de parturição, aplicando esforços para que flua naturalmente sem necessidade de intervenções dispensáveis, e investindo na construção da relação empática com a mulher e sua família desde o pré-natal até o puerpério (5). Desta forma, desde 1998, o procedimento "parto normal sem distócia realizado por enfermeiro obstetra" está incluído na tabela de Sistema de Informações Hospitalares do Sistema Único de Saúde e, a partir de 1999, o MS também propôs a criação de Centros de Parto Normal para atuação exclusiva do enfermeiro obstétrico(4).

Com o grande estímulo nacional da Rede Cegonha para a formação de enfermeiros obstétricos, o Conselho Federal de Enfermagem lançou em 2016 uma resolução específica que normatizou detalhadamente a atuação e a formação do enfermeiro obstétrico no âmbito do pré-natal, parto e puerpério(7).

Diante desse contexto, o objetivo deste estudo foi analisar as contribuições da enfermagem obstétrica para as boas práticas no trabalho de parto e parto vaginal.

\section{METODOLOGIA}

\section{Tipo de estudo}

Trata-se de um estudo transversal, descritivo, com abordagem quantitativa e retrospectiva.

\section{Participantes da pesquisa}

A amostra foi composta por 475 prontuários de mulheres que tiveram parto vaginal no hospital entre janeiro e dezembro de 2016, dos quais 94 resultaram de atendimentos por preceptores e enfermeiros residentes em obstetrícia, e 381 por outros profissionais (médicos obstetras, residentes em ginecologia e obstetrícia e/ou acadêmicos de medicina). A diferença do quantitativo de partos realizados entre enfermeiros e outros profissionais ocorreu devido à inexistência de serviço de Enfermagem Obstétrica implantado na instituição, sendo que os partos realizados pela equipe de Enfermagem se resumiram àqueles acompanhados exclusivamente pela equipe do Programa de Residência de Enfermagem Obstétrica.

Utilizou-se os seguintes critérios de inclusão: prontuários de mulheres que tiveram parto vaginal no hospital em estudo, com idade gestacional a partir de 37 semanas, e gestação de risco habitual, independentemente da paridade (de acordo com o protocolo institucional de atuação da Enfermagem Obstétrica). Foram excluidos aqueles prontuários com falhas nos registros de número de prontuários e no arquivamento, de mulheres que tiveram cirurgia cesárea e/ou que atenderam aos critérios de gestação de alto risco contemplados pelo MS (8).

\section{Local da pesquisa}

O cenário da pesquisa foi um hospital estadual referência em gestação de alto risco no estado de Goiás.

\section{Coleta dos dados}

A coleta de dados foi realizada de abril a setembro de 2018. Foram identificados 627 partos com idade gestacional igual ou maior que 37 semanas, porém houve falha no arquivamento de 46 prontuários e 106 não atenderam aos critérios de inclusão.

A escolha pelo periodo foi determinada a partir do mês em que os enfermeiros residentes em obstetrícia iniciaram o acompanhamento de partos na instituição. À época, apesar do perfil da instituição ser voltado ao atendimento de gestantes de alto risco, todas as gestantes que procuravam o serviço eram atendidas, de forma que o número de partos de risco habitual mantinha-se elevado.

Os dados foram transcritos para um questionário estruturado, desenvolvido especificamente para aplicação neste estudo, e construído a partir de revisão da literatura 
referente ao tema.

O questionário contemplou variáveis sociodemográficas (faixa etária, município de residência, cor, escolaridade, situação conjugal e atividade remunerada), clínicoobstétricas (paridade, número de consultas de pré-natal, uso de drogas lícitas e ilícitas), boas práticas no parto e nascimento (alimentação, métodos não farmacológicos para alivio da dor, partograma, presença de acompanhante no parto, contato pele a pele imediato, clampeamento do cordão e amamentação na primeira hora), e intervenções obstétricas (ocitocina, uso contínuo de fluidos intravenosos durante o trabalho de parto, amniotomia, posição de parto, episiotomia, e pressão no fundo do útero).

\section{Procedimentos de análise dos dados}

Para organização e tabulação, as informações foram transcritas no programa Microsoft Office Excel@ 2017. Os dados quantitativos foram analisados descritivamente, por meio de distribuição de frequências, médias, percentual e desvio padrão. Aplicaram-se os testes de qui-quadrado de Yates e Exato de Fisher para análise estatística, sendo consideradas estatisticamente significantes as diferenças em que $p$ foi menor que $5 \%(p<0,05)$. Na apresentação dos resultados, os totais variam devido a exclusão de dados sem informação.

\section{Procedimentos éticos}

O projeto foi aprovado pelo Comitê de Ética em Pesquisa via Plataforma Brasil, com número do parecer 2.616.944, CAAE: 81573317.3.0000.5080, conforme as recomendações propostas pelo Conselho Nacional de Saúde, por meio da Resolução 466/2012, que apresenta as diretrizes e normas regulamentadoras de pesquisas envolvendo seres humanos (9).

\section{RESULTADOS}

Em relação à caracterização do perfil sociodemográfico das mulheres que tiveram parto vaginal na instituição, verificou-se que a maioria tinha idade entre 18 e 35 anos $(78,7 \%)$ com média de idade de 22,7 anos $\pm 5,7$, era proveniente de municípios do interior de Goiás (64,3\%), de cor parda (75,3\%), sem companheiro (55,3\%), com 8 ou mais anos de estudo $(76,5 \%)$ e que não exercia atividade remunerada (71,3\%). Quanto ao perfil clínico-obstétrico das mulheres, a maioria delas era multípara (60,8\%), com 6 ou mais consultas de pré-natal (65,6\%), e que não fez uso de nenhum tipo de droga durante a gestação (85,7\%).

Em relação à realização das boas práticas aplicadas no trabalho de parto e parto, houve associação estatisticamente significante entre os partos sem os enfermeiros residentes em obstetrícia e a não utilização dos métodos não farmacológicos para alívio da dor ( $p<0.0000001)$, a ausência de utilização do partograma ( $p<0.0000001)$, a ausência de acompanhante no momento do parto $(p<0.0000001)$, o clampeamento precoce do cordão umbilical ( $p=0.00004323)$, e a privação da amamentação na primeira hora de vida do recém-nascido $(p=0.0001509)$ (Tabela 1).

Tabela 1. Boas práticas no trabalho de parto e parto de mulheres que tiveram parto vaginal num hospital estadual da região Central do Brasil no ano de 2016. Goiânia, Goiás, Brasil, 2018.

\begin{tabular}{|c|c|c|c|c|c|}
\hline \multirow[t]{2}{*}{$\begin{array}{l}\text { Boas prá- } \\
\text { ticas }\end{array}$} & \multicolumn{2}{|c|}{$\begin{array}{l}\text { Partos as- } \\
\text { sistidos pela } \\
\text { Enfermagem } \\
\text { Obstétrica }\end{array}$} & \multicolumn{2}{|c|}{$\begin{array}{l}\text { Partos assisti- } \\
\text { dos por outros } \\
\text { profissionais }\end{array}$} & \multirow[t]{2}{*}{ Valor de $p \neq$} \\
\hline & $\mathrm{n}^{\mathrm{a}}$ & $\%$ & $n^{a}$ & $\%$ & \\
\hline \multicolumn{5}{|c|}{ Alimentação } & \multirow{3}{*}{$0.3302^{*}$} \\
\hline Sim & 58 & 68,2 & 212 & 61,8 & \\
\hline Não & 27 & 31,8 & 131 & 38,2 & \\
\hline \multicolumn{5}{|c|}{ Métodos não farmacológicos para alívio da dor } & \multirow{3}{*}{$<0.0000001$} \\
\hline Sim & 52 & 74,3 & 32 & 14,0 & \\
\hline Não & 18 & 25,7 & 196 & 86,0 & \\
\hline \multicolumn{5}{|c|}{ Partograma } & \multirow{3}{*}{$<0.0000001^{*}$} \\
\hline Sim & 57 & 60,6 & 91 & 24,1 & \\
\hline Não & 37 & 39,4 & 287 & 75,9 & \\
\hline \multicolumn{5}{|c|}{ Presença de acompanhante no parto } & \multirow{3}{*}{$<0.0000001^{\star *}$} \\
\hline Sim & 16 & 17,2 & 3 & 0,8 & \\
\hline Não & 77 & 82,8 & 363 & 99,2 & \\
\hline \multicolumn{5}{|c|}{ Contato pele a pele imediato } & \multirow{3}{*}{$0.1050^{*}$} \\
\hline Sim & 36 & 72,0 & 26 & 54,2 & \\
\hline Não & 14 & 28,0 & 22 & 45,8 & \\
\hline \multicolumn{5}{|c|}{ Clampeamento do cordão } & \multirow{3}{*}{$0.00004323^{\star \star}$} \\
\hline Tardio & 30 & 36,1 & 38 & 14,7 & \\
\hline Precoce & 53 & 63,9 & 220 & 85,3 & \\
\hline \multicolumn{5}{|c|}{ Amamentação na la hora } & \multirow{3}{*}{$0.0001509^{*}$} \\
\hline Sim & 19 & 36,5 & 8 & 9,0 & \\
\hline Não & 33 & 63,5 & 81 & 91,0 & \\
\hline
\end{tabular}

Fonte: Dados da pesquisa. n: número de casos. \%: porcentagem.

${ }^{a}$ O n amostral não inclui os valores não informados

\# Os cálculos para o valor de $p$ não inclui os dados não informados

* Qui-quadrado corrigido de Yates

** Exato de Fischer

Já em relação às intervenções obstétricas, os partos assistidos por enfermeiros residentes em obstetrícia mantiveram-se associados à não realização da episiotomia $(p<0.0000001)$ (Tabela 2). 
Tabela 2. Intervenções obstétricas realizadas no trabalho de parto e parto de mulheres que tiveram parto vaginal num hospital estadual da região Central do Brasil no ano de 2016. Goiânia, Goiás, Brasil, 2018

\begin{tabular}{|c|c|c|c|c|c|}
\hline \multirow[t]{2}{*}{$\begin{array}{l}\text { Inter- } \\
\text { venções } \\
\text { Obstétri- } \\
\text { cas }\end{array}$} & \multicolumn{2}{|c|}{$\begin{array}{l}\text { Partos assistidos } \\
\text { pela Enfermagem } \\
\text { Obstétrcia }\end{array}$} & \multicolumn{2}{|c|}{$\begin{array}{l}\text { Partos assistidos por } \\
\text { outros profissionais }\end{array}$} & \multirow[t]{2}{*}{ Valor de $p \neq$} \\
\hline & $n^{a}$ & $\%$ & $n^{a}$ & $\%$ & \\
\hline \multicolumn{5}{|c|}{ Ocitocina } & \multirow{3}{*}{$0.1300^{*}$} \\
\hline Sim & 23 & 24,5 & 125 & 33,2 & \\
\hline Não & 71 & 75,5 & 251 & 66,8 & \\
\hline \multicolumn{5}{|c|}{$\begin{array}{l}\text { Uso continuo de flúidos intravenosos durante o } \\
\text { trabalho de parto }\end{array}$} & \multirow{3}{*}{$0.2607^{\star}$} \\
\hline Sim & 32 & 34,0 & 154 & 41,1 & \\
\hline Não & 62 & 66,0 & 221 & 58,9 & \\
\hline \multicolumn{5}{|c|}{ Amniotomia } & \multirow{3}{*}{$0.06183^{*}$} \\
\hline Sim & 9 & 15,0 & 48 & 28,2 & \\
\hline Não & 51 & 85,0 & 122 & 71,8 & \\
\hline \multicolumn{5}{|c|}{ Posição de parto } & \multirow{4}{*}{$0.3941^{* *}$} \\
\hline $\begin{array}{l}\text { Lito- } \\
\text { tômica }\end{array}$ & 91 & 96,8 & 376 & 98,7 & \\
\hline $\begin{array}{l}\text { De- } \\
\text { cúbito } \\
\text { dorsal }\end{array}$ & 3 & 3,2 & 4 & 1,0 & \\
\hline $\begin{array}{l}\text { De- } \\
\text { cúbito } \\
\text { lateral }\end{array}$ & 0 & 0 & 1 & 0,3 & \\
\hline \multicolumn{5}{|c|}{ Episiotomia } & \multirow{3}{*}{$<0.0000001^{*}$} \\
\hline Sim & 7 & 7,5 & 157 & 42,0 & \\
\hline Não & 86 & 92,5 & 217 & 58,0 & \\
\hline \multicolumn{5}{|c|}{ Pressão no fundo do útero } & \multirow{3}{*}{$0.6282^{\star *}$} \\
\hline $\operatorname{Sim}$ & 5 & 8,5 & 3 & 4,7 & \\
\hline Não & 54 & 91.5 & 61 & 95,3 & \\
\hline
\end{tabular}

Fonte: Dados da pesquisa. n: número de casos. \%: porcentagem.

a $\mathrm{O}$ amostral não inclui os valores não informados

‡ Os cálculos para o valor de $p$ não inclui os dados não informados

* Qui-quadrado corrigido de Yates

** Exato de Fischer

\section{DISCUSSÃO}

A presente pesquisa evidenciou que os partos assistidos pela Enfermagem Obstétrica são dotados de maior quantidade de boas práticas e possuem menos intervenções, enfatizando assim a importância da atuação deste profissional no trabalho de parto e parto vaginal de risco habitual. Assim, é possivel afirmar que a Enfermagem Obstétrica vem contribuindo e participando de forma efetiva para a adesão dos princípios atuais nacionais e internacionais preconizados para atenção humanizada ao parto e nascimento(10,11).
Nesse estudo, em relação ao uso das boas práticas e intervenções no trabalho de parto e parto vaginal, a diferença encontrada entre os partos assistidos pelos enfermeiros residentes em obstetrícia daqueles não assistidos por eles sugere que a atuação autônoma da Enfermagem Obstétrica contribui como ator facilitador para gerar experiência agradável em relação ao processo de parturição à mulher e sua família e também para a redução de intervenções não oportunas, demonstrando o diferencial do cuidado de Enfermagem.

A literatura ratifica que a Enfermagem Obstétrica vem se destacando pela atuação humanizada no parto, reduzindo a morbimortalidade materno-infantil e proporcionando experiência positiva à mulher e sua família(12).

Nesse estudo, houve baixa adesão por parte de outros profissionais à utilização dos métodos não farmacológicos para alívio da dor no trabalho de parto $(p<0.0000001)$, sendo essa boa prática mais presente nos partos acompanhados pela Enfermagem Obstétrica (74,3\%). Essa estratégia oferece conforto à mulher e contribui para uma experiência mais agradável do processo de parturição(13,14). Resultados de uma pesquisa realizada em Belo Horizonte corroboraram os deste estudo em relação aos profissionais de Enfermagem, apontando que $85 \%$ deles fizeram uso da medida ${ }^{(15)}$. $O$ fato de que a Enfermagem Obstétrica lança mão dessa estratégia com mais frequência sugere que a atuação diligente da classe contribui como fator facilitador para uma vivência menos traumática do trabalho de parto, entendendo que esses métodos fazem parte do cuidado obstétrico fornecido por essa categoria profissional, embora não sejam restritos a ela (15).

Nessa pesquisa, o partograma foi menos utilizado pelos outros profissionais $(p<0.0000001)$ e mais comumente presente entre os trabalhos de parto acompanhados pelos enfermeiros residentes em obstetrícia (60,6\%). Um estudo realizado em Luanda - África, com 40 profissionais de Enfermagem que atendiam ao parto vaginal, revelou que $100 \%$ deles faziam o uso rotineiro do preenchimento do partograma e compreendiam sua importância(16). O partograma é um instrumento para acompanhamento da evolução do trabalho de parto, o qual deve ser utilizado pelos profissionais de saúde para monitorar o bem-estar materno e fetal e identificar riscos para desfechos adversos ${ }^{(11)}$

$\mathrm{Na}$ presente casuística, os partos assistidos sem a presença dos enfermeiros residentes em obstetrícia foram associados à ausência de acompanhante durante o parto $(p<0.0000001)$. Apesar desse direito ser garantido por lei, há mais de dez anos, ainda existe desconhecimento por parte das parturientes, como relata o estudo realizado em Sergipe com 160 gestantes, o qual confirmou o fato por 
parte de $57,5 \%$ das parturientes, e também revelou que $66,2 \%$ não desfrutaram da presença do acompanhante em todo o processo de parturição, como garante a lei e conforme deveria ser cumprido pelas maternidades ${ }^{(17)}$. A Organização Mundial da Saúde recomenda um acompanhante da escolha da mulher durante todo o processo parturitivo para uma experiência positiva em relação ao parto, pois essa pessoa será capaz de promover suporte contínuo à parturiente de forma a compreender melhor suas necessidades ${ }^{(11)}$.

Nessa pesquisa, em relação à secção do cordão umbilical, os partos não assistidos pela Enfermagem Obstétrica apresentaram clampeamento precoce $(p=0.00004323)$. $O$ atraso no clampeamento do cordão beneficia o RN, pois, aumenta em até $50 \%$ as reservas de ferro até os seis meses de idade, propicia o contato pele a pele imediato, e para os prematuros reduz os riscos de hemorragia intraventricular, enterocolite necrosante e chances de transfusão sanguínea, podendo ser realizado também em cirurgias cesáreas ${ }^{(18-20,11)}$. Portanto, se o bebê de risco habitual apresenta boa vitalidade ao nascer, independentemente da cor do líquido amniótico, o clampemanto tardio é indicado(21).

A prática da amamentação precoce não esteve presente em nascimentos assistidos por profissionais não enfermeiros $(p=0.0001509)$. Pesquisa realizada no Paraná identificou ausência de implementação dessa boa prática na instituição pesquisada e deficiência de conhecimento profissional relacionado ao aleitamento materno na primeira hora de vida do RN (22). Todos os RNs, até mesmo os de baixo peso, que são capazes de sugar devem ser colocados junto ao seio materno, estimulados e auxiliados a amamentar o quanto antes, logo após o nascimento ${ }^{(23,11)}$. Estudo realizado no Rio de Janeiro em 2015 com 367 parturientes assistidas por enfermeiras obstétricas constatou que o aleitamento materno na primeira hora de vida foi realizado em $82 \%$ dos nascimentos ${ }^{(24)}$, demostrando que a Enfermagem Obstétrica emprega maior empenho na aplicação das boas práticas, obedecendo às recomendações mundiais e apresentando preocupação em oferecer o melhor cuidado possivel ao binômio mãe-bebê.

Este estudo apontou um papel importante do modelo assistencial pouco intervencionista em relação à prevalência de episiotomia, sendo que o parto vaginal assistido por enfermeiros residentes em obstetrícia esteve associado à não realização desta intervenção ( $p<0.0000001)$. No Brasil, estudo realizado com 655 mulheres resultou em uma taxa de 16,1\% de episiotomias realizadas por profissionais de Enfermagem contra $85,2 \%$ realizadas por outros profissionais ${ }^{(15)}$. As recomendações mundiais desestimulam o uso rotineiro de episiotomia, afirmando que não existem evidências científicas suficientemente eficazes que defendam seu uso em nenhum cenário ou circunstância específica ${ }^{(11)}$.
Uma Revisão Sistemática Cochrane mostrou que alguns profissionais acreditam que o uso rotineiro da episiotomia pode prevenir lacerações perineais extensas durante o parto. Contudo, a realização da episiotomia torna-se uma garantia de trauma perineal e suturas, além de poder resultar também em aumento do risco de lacerações perineais extensas, mulheres com dor moderada ou severa, dispareunia a longo prazo e incontinência urinária(25)

\section{Limitações do estudo}

Este estudo apresentou como limitação a coleta de dados secundários, entendendo que algumas informações não foram encontradas nos prontuários por falha no arquivamento e/ou ausência de preenchimento adequado do partograma, sendo que alguns aspectos abordados poderiam ser obtidos com maior fidedignidade por meio de entrevista com a puérpera, ou até um acompanhamento prospectivo. Esses fatos podem ter influenciado os resultados, embora as informações encontradas apontem para desfecho difundido pelas publicações da área.

\section{Contribuições do estudo para a prática}

Os resultados desta pesquisa ratificam as orientações mundiais e nacionais, as quais associam o maior uso das boas práticas do parto e nascimento à atuação assistencial de categorias profissionais não médicas, entendendo que os médicos são melhor treinados para o uso de tecnologias específicas focadas na alta complexidade e que os enfermeiros têm sua formação voltada para o cuidar, privilegiando a atuação humanizada(6,11).

A prática humanizada e também a inserção do enfermeiro obstétrico no atendimento à gestante apresentam vantagens em relação à redução de intervenções e maior satisfação das mulheres. A residência em Enfermagem Obstétrica no Brasil tem como finalidade formar profissionais bem qualificados e sensiveis aos princípios norteadores da atenção à saúde da mulher e do neonato, preconizados pelo MS, com vistas a intensificar a assistência integral qualificada e baseada em evidências científicas atualizadas, além de colaborar para uma experiência positiva no parto ${ }^{(7,10)}$.

\section{CONCLUSÃO}

O presente estudo reflete a realidade assistencial do Sistema Único de Saúde na região central do país através de um corte populacional de mulheres atendidas em um hospital de referência para o estado de Goiás e o quão beneficiadas estão em relação às boas práticas de atenção ao parto humanizado e menos expostas às práticas intervencionistas, sem real indicação, quando atendidas pela Enfermagem Obstétrica. 
Essa evidência pode colaborar com o sustento às políticas atuais de saúde da mulher e da criança, e oferecer um subsidio científico para a instituição que sediou a pesquisa, bem como para outros hospitais-escola e maternidades passarem a investirna formação e contratação de enfermeiros obstétricos para dar continuidade à assistência de boa qualidade, com garantia do uso de boas práticas, gerando bons resultados nos indicadores de qualidade do cuidado obstétrico.

É importante também salientar que as intervenções obstétricas ainda estão presentes e que as boas práticas no parto e nascimento não são realidades para todas as mulheres. Assim, cabe às instituições de saúde e aos seus profissionais repensarem a forma de atuação, relembrando que o parto é um evento fisiológico que geralmente não necessitará de muitas interferências por parte da equipe de saúde, e entendendo que o contexto de saúde maternoinfantil caminha para a redução da morbimortalidade em direção à qualidade da assistência e à experiência satisfatória em relação ao parto vaginal e nascimento.

Os resultados desta pesquisa abrangeram apenas um hospital da região central do país, no entanto, seus achados conferem importância ao tema. Portanto mais estudos para o cenário nacional são necessários, pois poderiam proporcionar uma melhor avaliação do modelo obstétrico difundido, para que novas estratégias de transformação das práticas obstétricas sejam levantadas.

Agradecemos à instituição hospitalar que sediou a pesquisa e também ao Programa de Residência Uniprofissional de Enfermagem Obstétrica da Secretaria Estadual de Saúde de Goiás. Este estudo foi financiado pelo próprio pesquisador. Não houve conflitos de interesse entre os autores.

\section{Contribuição dos autores}

Concepção e/ou desenho da pesquisa: Taynara Cassimiro de Moura Alves, Marília Cordeiro de Sousa e Priscila Salomão da Silva; Análise e interpretação dos dados: Marília Cordeiro de Sousa, Taynara Cassimiro de Moura Alves, Amanda Santos Fernandes Coelho e Nayara Franklin Cesar; Redação do artigo: Taynara Cassimiro de Moura Alves e Marilia Cordeiro de Sousa; Revisão crítica: Amanda Santos Fernandes Coelho, Leonora Rezende Pacheco, e Marília Cordeiro de Sousa Revisão final: Amanda Santos Fernandes Coelho, Taynara Cassimiro de Moura Alves, Marília Cordeiro de Sousa e Nayara Franklin Cesar. 


\section{REFERÊNCIAS}

1. Silva FL, Souza ALS, Leite-Salgueiro CDB, Barbosa LS, Lobato L, Pereira JS. O protagonismo do enfermeiro na Política Nacional de Humanização do Parto e do Nascimento e com vistas a erradicação de abuso e violência obstétrica. Id on Line Rev. Mult. Psic. [internet]. 2018 [cited 2019 jan 21]:12(41):1068-1082. Avaliable from: https://idonline.emnuvens.com.br/ id/article/view/1265/1874

2. World Health Organization. Care in normal birth: A practical guide. Birth Issues in Perinatal Care [internet]. 1997 [cited 2019 jan 23]:24(2):121-123. Avaliable from: https://www.ncbi.nlm.nih.gov/pubmed/9271979.

3. Ministério da Saúde (BR). Programa de humanização no pré-natal e nascimento. Braília: Ministério da Saúde [internet]. 2002. [cited 2019 jan 23]. Avaliable from: http://bvsms.saude.gov.br/bvs/publicacoes/parto. pdf.

4. Ministério da Saúde (BR). Cadernos HumanizaSUS: Humanização do parto e do nascimento. Brasilia: Ministério da Saúde [internet]. 2014 [cited 2019 jan 23]. Avaliable from: http://www.redehumanizasus.net/sites/ default/files/caderno_humanizasus_v4_humanizacao_parto.pdf.

5. Duarte MR, Alves VH, Rodrigues DP, Souza KV, Pereira AV, Pimentel MM. Tecnologias do cuidado na enfermagem obstétrica: contribuição para o parto e nascimento. Cogitare enferm. [internet]. 2019 [cited 2019 jan 21];24(e54164):1-11. Avaliable from: http://dx.doi.org/10.5380/ ce.v24i0.54164.

6. Brasil. Ministério da Saúde (BR). Portaria n으 1.459, de 24 de junho de 201 [Institui, no âmbito do Sistema Único de Saúde - SUS - a Rede Cegonha] Diário Oficial [da] República Federativa do Brasil. [internet]. 2011 [cited 2018 nov ll]. Avaliable from: http://bvsms.saude.gov.br/bvs/saudelegis/ gm/2011/prt1459_24_06_2011.html.

7. Conselho Federal de Enfermagem (BR). Resolução no 0516/2016 Brasilia: Conselho Federal de Enfermagem [internet]. 2016 [cited 2018 nov 11]. Avaliable from: http://www.cofen.gov.br/resolucao-cofenno-05162016_41989.html.

8. Ministério da Saúde (BR). Gestação de Alto Risco: Manual técnico. 5 a ed. Brasilia: Ministério da Saúde [internet]. 2010 [cited 2019 jan 23]. Avaliable from: http://bvsms.saude.gov.br/bvs/publicacoes/manual_ tecnico_gestacao_alto_risco.pdf.

9. Brasil. Ministério da Saúde (BR). Resolução n‥ 466, de 12 de dezembro de 2012 [Diretrizes e normas regulamentadoras de pesquisas envolvendo seres humanos]. Diário Oficial [da] República Federativa do Brasi [internet]. 2012 [cited 2018 nov 11]. Avaliable from: http://bvsms.saude. gov.br/bvs/saudelegis/cns/2013/res0466_12_12_2012.html.

10. Comissão Nacional de Incorporação de Tecnologias no SUS (BR) Diretrizes nacionais de assistência ao parto: Relatório de recomendação. Brasilia: Ministério da Saúde (211) [internet]. 2017 [cited 2019 jan 23]. Avaliable from: http://conitec.gov.br/images/Consultas/2016/Relatorio_ Diretriz-PartoNormal_CP.pdf.

11. World Health Organization. Who recommendations: Intrapartum care for a positive childbirth experience. Geneva: World Health Organization [internet]. 2018 [cited 2019 jan 23]. Avaliable from: https://www.who.int/ reproductivehealth/publications/intrapartum-care-guidelines/en/.

12. Reis CSC, Souza DOM, Nogueira MFH, Progianti JM, Vargens OMC Análise de partos acompanhados por enfermeiras obstétricas na perspectiva da humanização do parto e nascimento. J. res.: fundam. care. online [internet]. 2016 [cited 2019 jan 23];8(4):4972-4979. Avaliable from: http://www.seer.unirio.br/index.php/cuidadofundamental/article/ view/3966/pdf_1.

13. Hanum SP, Mattos DV, Matão MEL, Martins CA. Estratégias não farmacológicas para o alivio da dor no trabalho de parto: efetividade sob a ótica da parturiente. Rev enferm UFPE on line [internet]. 2019 [cited 2019 jan 23]:11(8):3303-3309. Avaliable from: https://periodicos.ufpe.br/ revistas/revistaenfermagem/article/viewFile/110197/22089.
14. Dias EG, Ferreira ARM, Martins AMC, Jesus MM, Alves JCS. Eficiência de métodos não farmacológicos para alivio da dor no trabalho de parto normal. Enferm Foco [internet]. 2018 [cited 2019 jan 21];9(2):3539. Avaliable from: http://revista.cofen.gov.br/index.php/enfermagem/ article/view/1398/442.

15. Vogt SE, Silva KS, Dias MAB. Comparação de modelos de assistência ao parto em hospitais públicos. Rev Saúde Pública [internet]. 2014 [cited 2019 jan 23]:48(2):304-313. Avaliable from: http://www.scielo.br/scielo. php?pid=S0034-89102014000200304Escript=sci_abstractEtlng=pt.

16. Alexandre DFSN, Mamede FV, Pudêncio PS. O uso do partograma por profissionais de enfermagem durante o trabalho de parto. Enfermagem Obstétrica [internet]. 2016 [cited 2019 jan 21];3(34):1-6. Avaliable from: http://www.enfo.com.br/ojs/index.php/EnfObst/article/view/34/33.

17. Santos ECP, Lima MR, Conceição LL, Tavares CS, Guimarães AMDN. Conhecimento e aplicação do direito do acompanhante na gestação e parto. Enferm. foco [internet]. 2016 [cited 2019 jan 23]:7(3/4):61-65. Avaliable from: http://revista.cofen.gov.br/index.php/enfermagem/ article/view/918

18. Vitral GLN, Reis ZSN, Gaspar JS, Souza IMF, Aguiar RALP. Clampeamento oportuno de cordão umbilical e suas repercussões na concentração de hemoglobina neonatal. SaBios [internet]. 2017 [cited 2019 feb 15]:11(3):35-41. Avaliable from: http://revista2.grupointegrado.br/ revista/index.php/sabios2/article/view/2062/968.

19. World Health Organization. Guideline: delayed umbilical cord clamping for improved maternal and infant health and nutrition outcomes. Geneva: World Health Organization [internet]. 2014 [cited 2019 jan 23]. Avaliable from: https://www.who.int/nutrition/publications/guidelines/cord_ clamping/en/.

20. Andersson O, Hellström-Westas L, Domellöf M. Elective caesarean: does delay in cord clamping for $30 \mathrm{~s}$ ensure sufficient iron stores at 4 months of age? A historical cohort control study. BMJ Open [internet] 2016 [cited 2019 jan 21];6(11):1-9. Avaliable from: https://www.ncbi.nlm.nih gov/pmc/articles/PMC5129052/

21. Sociedade Brasileira de Pediatria (BR). Reanimação do recémnascido $\geq 34$ semanas em sala de parto: Diretrizes 2016 da Sociedade Brasileira de Pediatria 26 de janeiro de 2016. São Paulo: Sociedade Brasileira de Pediatria [internet]. 2016 [cited 2019 jan 15]. Avaliable from: http://www.sbp.com.br/fileadmin/user_upload/ DiretrizesSBPReanimacaoRNMaior34semanas26jan2016.pdf.

22. Antunes MB, Demitto MO, Soares LG, Radovanovic CAT, Higarashi IH, Ichisato SMT et al. Amamentação na primeira hora de vida: conhecimento e prática da equipe multiprofissional. Ave. Enferm. [internet]. 2017 [cited 2019 jan 21]:35(1):19-29. Avaliable from: http://www.scielo.org.co/pdf/ aven/v35nl/v35nla03.pdf

23. Silva JLP, Linhares FMP, Barros AA, Souza AG, Alves DS, Andrade PON. Fatores associados ao aleitamento materno na primeira hora de vida em um Hospital Amigo da Criança. Texto Contexto Enferm [internet]. 2018 [cited 2019 feb 15];27(4):1-10. Avaliable from: http://www.scielo.br/pdf/ tce/v27n4/0104-0707-tce-27-04-e4190017.pdf.

24. Ramos WMA, Aguiar, BGC, Conrad D, Pinto CB, Mussumeci, PA Contribuição da enfermeira obstétrica nas boas práticas da assistência ao parto e nascimento. J. res.: fundam. care. online [internet]. 2018 [cited 2019 jan 23];10(1):173-179. Avaliable from: http://www.seer.unirio.br/index. php/cuidadofundamental/article/view/6019/pdf.

25. Jiang H, Oian X, Carroli G, Garner P. Selective versus routine use of episiotomy for vaginal birth: Review. Cochrane Database Syst Ver [internet]. 2017 [cited 2019 jan 21];2(2):1-77. Avaliable from: https://www. cochranelibrary.com/cdsr/doi/10.1002/14651858.CD000081.pub3/full. 